\title{
A climatology of infrasound detections in northern Norway at the experimental ARCI array
}

\author{
Läslo Gerardus Evers • Johannes Schweitzer
}

Received: 20 August 2009 / Accepted: 17 March 2011

(C) Springer Science+Business Media B.V. 2011

1 Abstract The study of infrasound is experiencing 2 a renaissance in recent years since it was chosen 3 as a verification technique for the Comprehen4 sive Nuclear-Test-Ban Treaty. Currently, 60 infra5 sound arrays are being installed to monitor the 6 atmosphere for nuclear tests as part of the Inter7 national Monitoring System (IMS). The number 8 of non-IMS arrays also increases worldwide. The 9 experimental ARCES infrasound array (ARCI) is 10 an example of such an initiative. The detectabil11 ity of infrasound differs for each array and is a 12 function of the array location and configuration, 13 the state of the atmosphere, and the presence of 14 natural and anthropogenic sources. In this study, 15 a year of infrasound data is analyzed as recorded 16 by ARCI. Contributions of the atmosphere and

L. G. Evers

Seismology Division, Royal Netherlands

Meteorological Institute, PO Box 201,

3730 AE De Bilt, The Netherlands

\section{G. Evers ( $\bowtie)$}

Acoustic Remote Sensing Group, Faculty of Aerospace Engineering, TU Delft, PO Box 5, 2600 AA Delft, The Netherlands e-mail: evers@knmi.nl

J. Schweitzer

NORSAR, PO Box 53 , 2027 Kjeller, Norway e-mail: johannes@norsar.no the sources are evaluated in both a low- (0.1- 17 $1.0 \mathrm{~Hz})$ and high-frequency $(1.0-7.0 \mathrm{~Hz})$ pass- 18 band. The enormous number of detections in the 19 low-frequency band is explained in terms of the 20 stratospheric wind and ocean wave activity and 21 compared with the detection of microseism. Un- 22 derstanding the detectability in the low-frequency 23 band is of utmost importance for successfully ap- 24 plying infrasound as a verification technique since 25 small-sized nuclear test will show up in this fre- 26 quency range.

Keywords Infrasound - Array • Signal detection • 28 Source identification $\cdot$ Acoustic propagation

Infrasound was first discovered after the violent 31 eruption of the Krakatoa, Indonesia, in 1883. 32 Low-frequency pressure waves were observed at 33 traditional barographs. These appeared to have 34 traveled with the sound speed and up to four pas- 35 sages where noticed at some instruments (Symons 36 1888). The first microbarometer recordings date 37 from 1908 when a comet, or asteroid, exploded 38 over Siberia in Russia, the so-called Tunguska 39 event (Whipple 1930). The societal and scientific 40 interest in infrasound increased during World War 41 I, e.g., Whipple (1939), and later on in the nuclear 42 testing era (Posey and Pierce 1971). With the 43 
44 signature of the Limited Test Ban Treaty in 1963, 45 most interest in infrasound promptly came to a 46 stop, since nuclear tests were confined to the sub47 surface. Only a few studies could be maintained 48 (Balachandran et al. 1977; Liszka 1978). In recent 49 years, the study of infrasound gained renewed 50 interest because of the Comprehensive Nuclear51 Test-Ban Treaty (CTBT) that opened for signing 52 in 1996, where it is used as a verification technique 53 for atmospheric tests (Dahlman et al. 2009).

54 Sources of infrasound are in general large, since 55 an enormous amount of air has to be displaced 56 to generate such low frequencies (Gossard and 57 Hooke 1975). Natural sources are avalanches, 58 lightning, meteors, oceanic waves, earthquakes, 59 severe weather, volcanoes, and sprites. Among 60 anthropogenic sources are explosions, supersonic 61 flights, military activity, rocket launches, and nu62 clear tests. Identifying the sources of infrasound 63 out of this zoo of coherent waves in the at- mosphere is one of the major challenges in infra- 64 sound research.

The propagation of infrasound through the 66 highly dynamic atmosphere plays an important 67 role in source identification. Infrasound travels 68 up to thermospheric altitudes of $120 \mathrm{~km}$ and ex- 69 periences refractions due to an increase in wind 70 and/or temperature as a function of altitude. 71 If the gradients in the propagation velocity are 72 strong enough, infrasound will be sent back to the 73 Earth's surface (Drob et al.2003). There are three 74 regions in the atmosphere where such gradients 75 might exist. These are of importance in long-range 76 sound propagation, i.e., over distances larger than 77 $150 \mathrm{~km}$. The regions are marked by (1) a strong jet 78 stream at $10 \mathrm{~km}$ altitude, near the tropopause; (2) 79 the combined effect of wind and temperature at 80 the stratopause, around $50 \mathrm{~km}$ altitude; and (3) the 81 temperature increase in the thermosphere from 82 $100 \mathrm{~km}$ and upward.

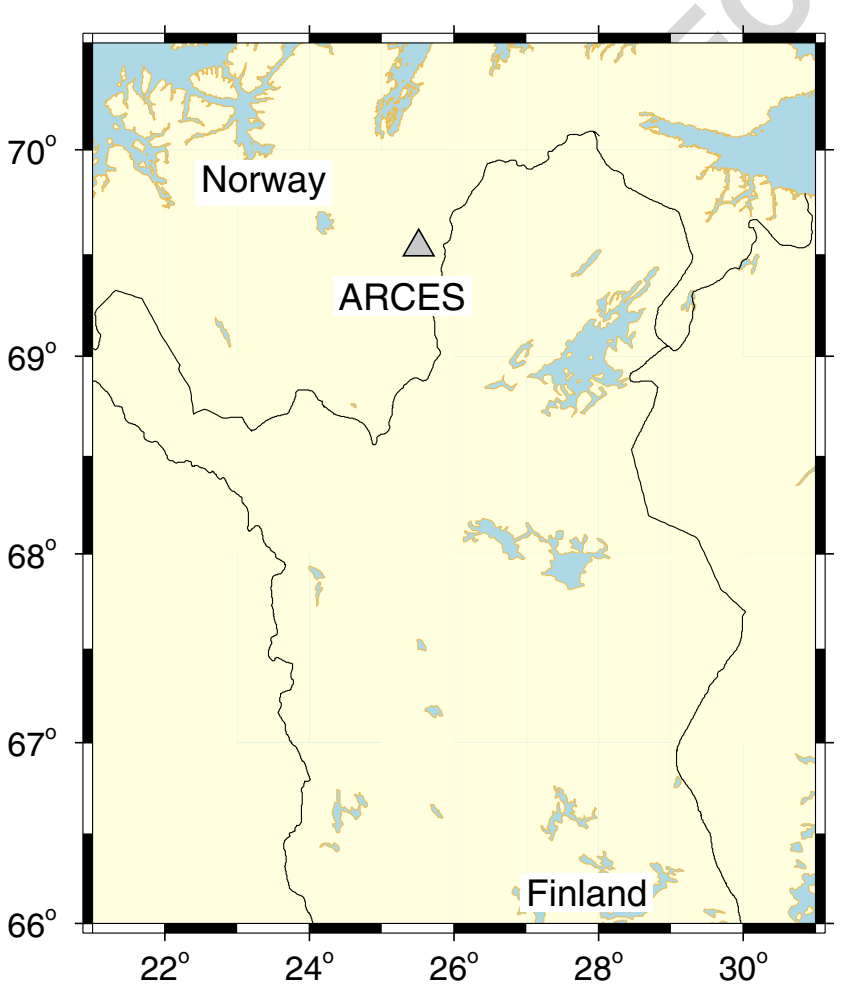

Fig. 1 The location of the ARCES seismic array and positions of the seismometers (gray dots). The temporary array ARCI is configured with three microbarometers (red

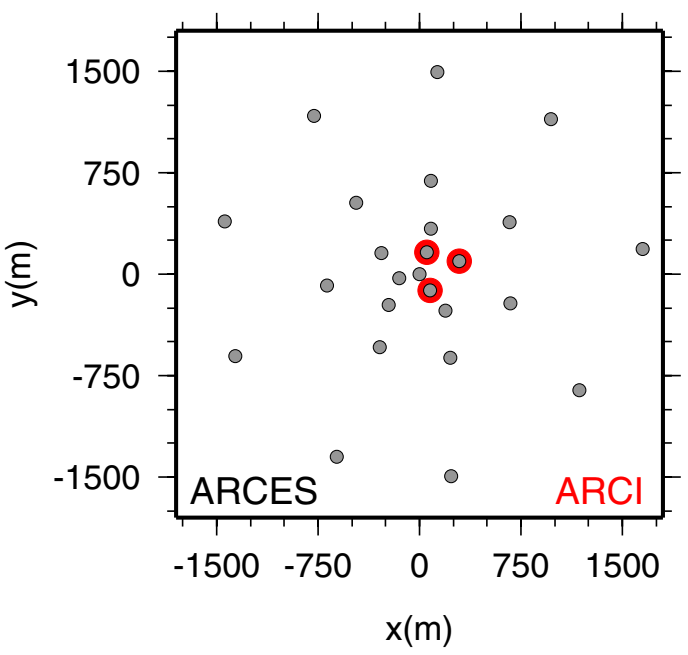

circles), which are co-located with seismometers in the center of the seismic array 
84 The aim of this study is to identify the sources 85 around the ARCES array and to build up a clima86 tology of station-specific detections. Each infra87 sound array has its own detection capabilities as 88 its configuration, the atmospheric conditions, and 89 source characteristics are highly variable as func90 tion of geographical location and time. Special 91 attention will be paid to the low-frequency band of 920.1 to $1.0 \mathrm{~Hz}$ which is of utmost importance for the 93 verification of the CTBT as small-sized nuclear 94 tests (around $1 \mathrm{kT} \mathrm{TNT}$ ) are expected to generate 95 infrasound of 0.1 to $0.2 \mathrm{~Hz}$ (Evers and Haak 2001). 96 It is also this band in which the almost continuous 97 background noise of microbaroms is present that 98 peak around $0.2 \mathrm{~Hz}$ (Posmentier 1967).

\section{The ARCES infrasound array}

100 A three-element experimental infrasound array 101 was established at ARCES in March 2008, which 102 will be abbreviated as ARCI (Roth et al. 2008). 103 The purpose of the installation is to gain ex104 perience with the simultaneous recording of in105 frasonic and seismological data. Figure 1 shows 106 the location and configuration of ARCI. The in107 struments are microbarometers of type Martec 108 MB2005 which have a flat frequency response to 109 pressure in the range of 0.01 to $27 \mathrm{~Hz}$. Infrasound 110 measurements are affected by noise due to wind. 111 Therefore, a spatial filter is applied at each in112 strument which essentially integrates the pressure 113 field. Doing so, pressure fluctuations with a small 114 coherency length, like those of tens of centimeters 115 associated with wind noise, are partly canceled 116 out. The infrasonic waves of interest remain undis117 turbed because of their much larger coherency 118 length of tens to hundreds of meters. Such analog 119 filters can consist of a pipe array with discrete 120 inlets or porous hoses (Hedlin et al 2003). The lat121 ter approach is applied at ARCI with four soaker 122 hoses, each with a length of $12 \mathrm{~m}$, connected to the 123 MB2005. For one of the three sites, the hoses are 124 additionally centered in a drainage pipe. Environ125 mental restrictions at the ARCES array prevent 126 the installation of larger pipe arrays that require 127 fencing. The applied noise reduction should be 128 considered as minimal. The atmospheric pressure changes around ARCI are sampled at a rate of 129 $80 \mathrm{~Hz}$.

\section{Data processing and signal detection}

\subsection{The approach}

The detections of coherent infrasonic signals trav- 133 eling over the array can be achieved by evaluating 134 the Fisher $(F)$ ratio. The $F$ detector has been 135 described in both the time (Melton and Bailey 136 1957) and frequency domain (Smart and Flinn 137 1971). In essence, a statistical hypothesis is tested. 138 Applying a $F$-detector is attractive because of its 139 well-known statistical distribution. The hypothesis 140 to be tested is that all recordings made by the mi- 141 crobarometers consist of uncorrelated noise. The 142 alternative hypothesis is valid for the case that not 143 only noise is present but also signal. Evaluated 144 are the variance of the noise and the variance 145 of all recordings, which cannot be attributed to 146 the noise since it is common to all recordings. 147 The $F$ detector has been successfully applied in 148 infrasound processing to detected, for example, 149 meteors and microbaroms (Evers and Haak 2001). 150 The processing sequence applied in this study is as 151 follows:

- Remove the mean of the recordings. 153

- Band-pass filter with a second-order Butter- 154 worth filter with corner frequencies of 0.1155 and $1.0 \mathrm{~Hz}$ (the low-frequency or microbarom 156 band) and 1.0 and 7.0 (the high-frequency 157 band).

- Decimate the data with a factor of 4, to re- 159 duce the data volume in order to minimize 160 the computational efforts, from a $80-$ to $20-\mathrm{Hz} 161$ sampling rate.

- Define a slowness grid between -0.005 and 163 $0.005 \mathrm{~s} / \mathrm{m}$ of $100 \times 100$ points, forming 10,000 164 beams.

- Split the data in segments of 256 samples, 166 which equals a bin of $12.8 \mathrm{~s}$.

- Evaluate the Fisher ratio for each beam in 168 each bin (with 50\% overlapping bins). 169

- Extract the slowness value, i.e., the backaz- 170 imuth and apparent sound speed, at the maxi- 171 mum Fisher ratio, for each bin. 


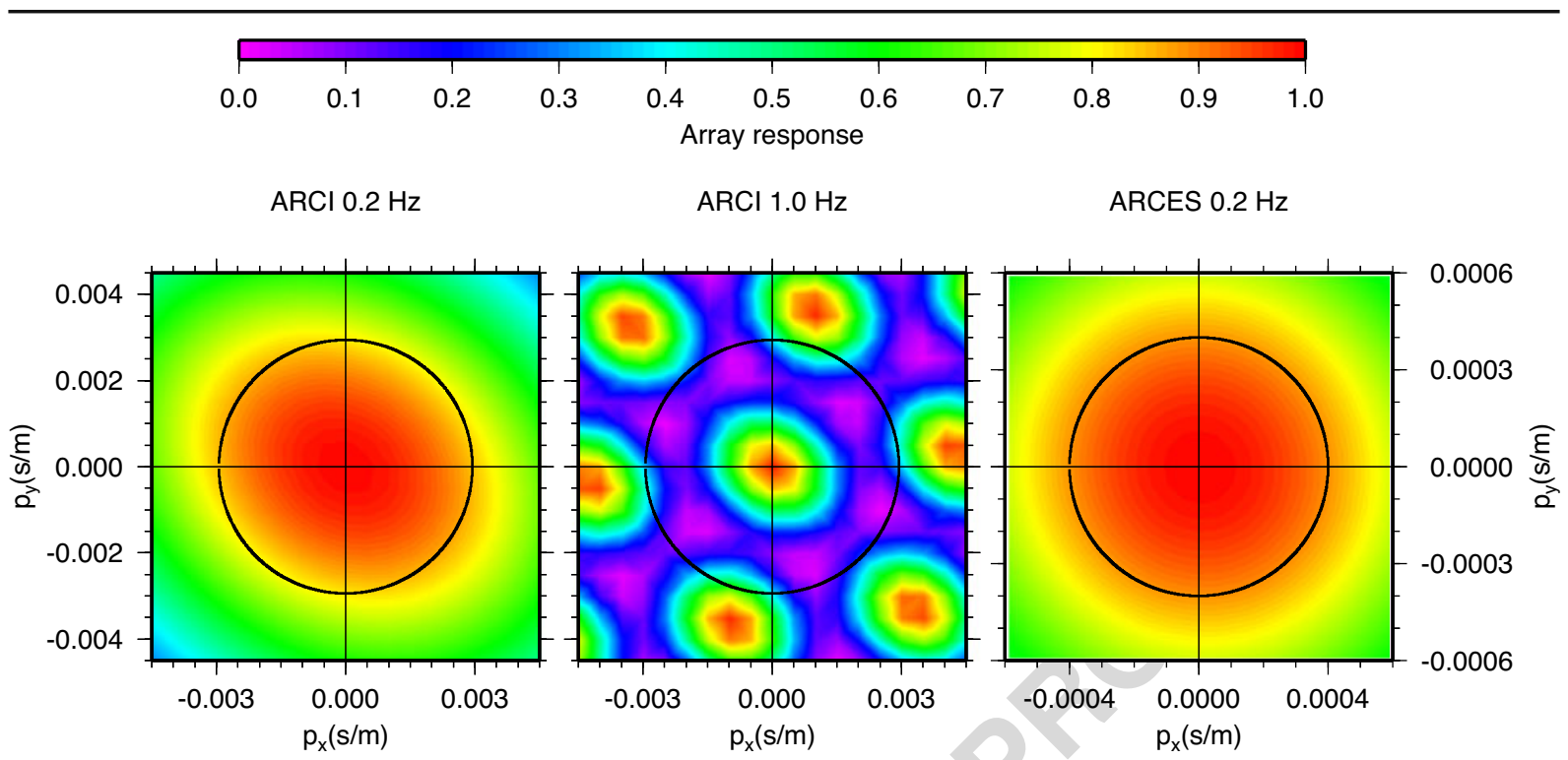

Fig. 2 The array response of ARCI to a $0.2-$ and $1.0-\mathrm{Hz}$ planar wave (left two frames). The black circle represent an apparent velocity of $340 \mathrm{~m} / \mathrm{s}$. The array response of

187 Most sources in the high-frequency band are man188 made. Figure 3 shows the time of occurrence of 189 events in this band, for the period of March 13, 1902008 up to May 14, 2009. There appear to be less 191 events during the weekends (days 6 and 7), com192 pared to weekdays, and during nighttime. In other 193 words, most events occur during the working week 194 and at daytime hours, which clearly indicates that 195 the sources are of anthropogenic origin. The re-
ARCES is given in the right frame at $0.2 \mathrm{~Hz}$; the black circle corresponds to an apparent velocity of $2,500 \mathrm{~m} / \mathrm{s}$

solved backazimuths with respect to ARCI are 196 given in the left frame of Fig. 4. Most events occur 197 from an eastern to southwestern direction. Some 198 of these can be explained by quarries, mines, and 199 military activity, as indicated by the red lines. 200 The source of the peak around $190^{\circ}$ has not yet 201 been identified. Less events find their origin in the 202 north, although two distinct peaks, around $290^{\circ} 203$ and $330^{\circ}$, indicate activity to the northwest. 204

3.3 Detections in the low-frequency band

Figure 5 shows the results of the previously de- 206 scribed processing approach for ARCI data in 207 the low-frequency band between 0.1 and $1.0 \mathrm{~Hz}$. 208 The lower frame shows the maximum Fisher ratio 209 for each bin. This value is related to the squared 210 signal-to-noise ratio (SNR) on the traces (see axis 211 on the right). The middle and top frames show 212 the resolved apparent sound speed and backaz- 213 imuth. Color coded are the number of detections 214 within an hour, where five or more detections 215 are denoted by red. Here, only detections with a 216 SNR larger than 1.0 are plotted, which equals a 217 Fisher ratio of four and higher. Such a detection 218 


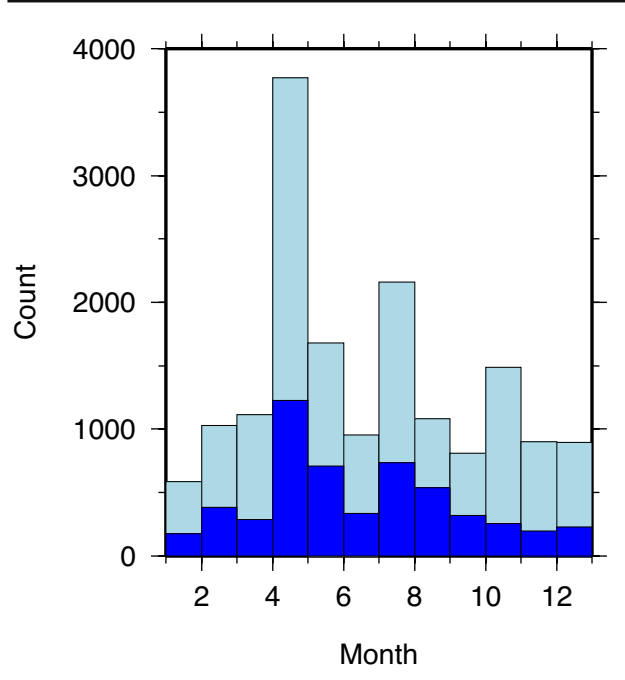

Fig. 3 Results from the array processing of ARCI data in the high-frequency band of 1.0 to $7.0 \mathrm{~Hz}$. The histograms shows the time of occurrence of infrasound events, between March 13, 2008 and May 14, 2009. Light blue colors indicate events with an signal-to-noise ratio (SNR) larger

219 will be labeled as an event and is mostly related 220 to microbarom activity. It follows from the lower 221 frame of Fig. 5 that signal coherency strongly 222 fluctuates as function of time. Large changes are seen from day to day, but there also seems to be a difference between winter and summertime (May to September). These are also reflected in the 225 resolved apparent sound speed and backazimuth. 226 The short time variations in signal coherency show 227 up as gaps, which means that no events have been 228 detected. During summer, less coherent events are 229 detected than in winter, and they appear from 230

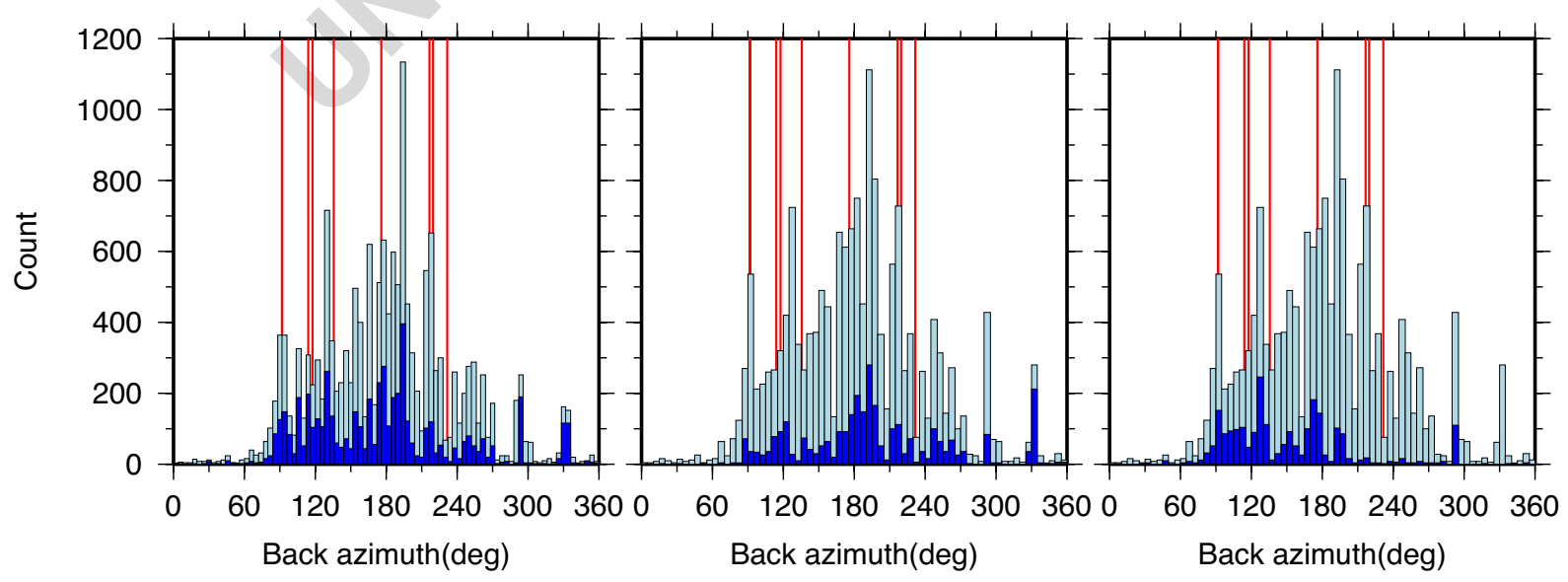

Fig. 4 The number of events (count) as function of the backazimuth for the high-frequency band. Events with a SNR larger than 1 are denoted by light blue; blue is used for a SNR larger than 1.5. The red lines give the backazimuths than 1 (or Fisher ratio of 4 and higher). Dark blue means a SNR larger than 1.5. The weekday diagram starts with day 1 which is Monday. For the hour histogram, local time in Norway is UTC $+2 \mathrm{~h}$ for summer and UTC $+1 \mathrm{~h}$ for winter 


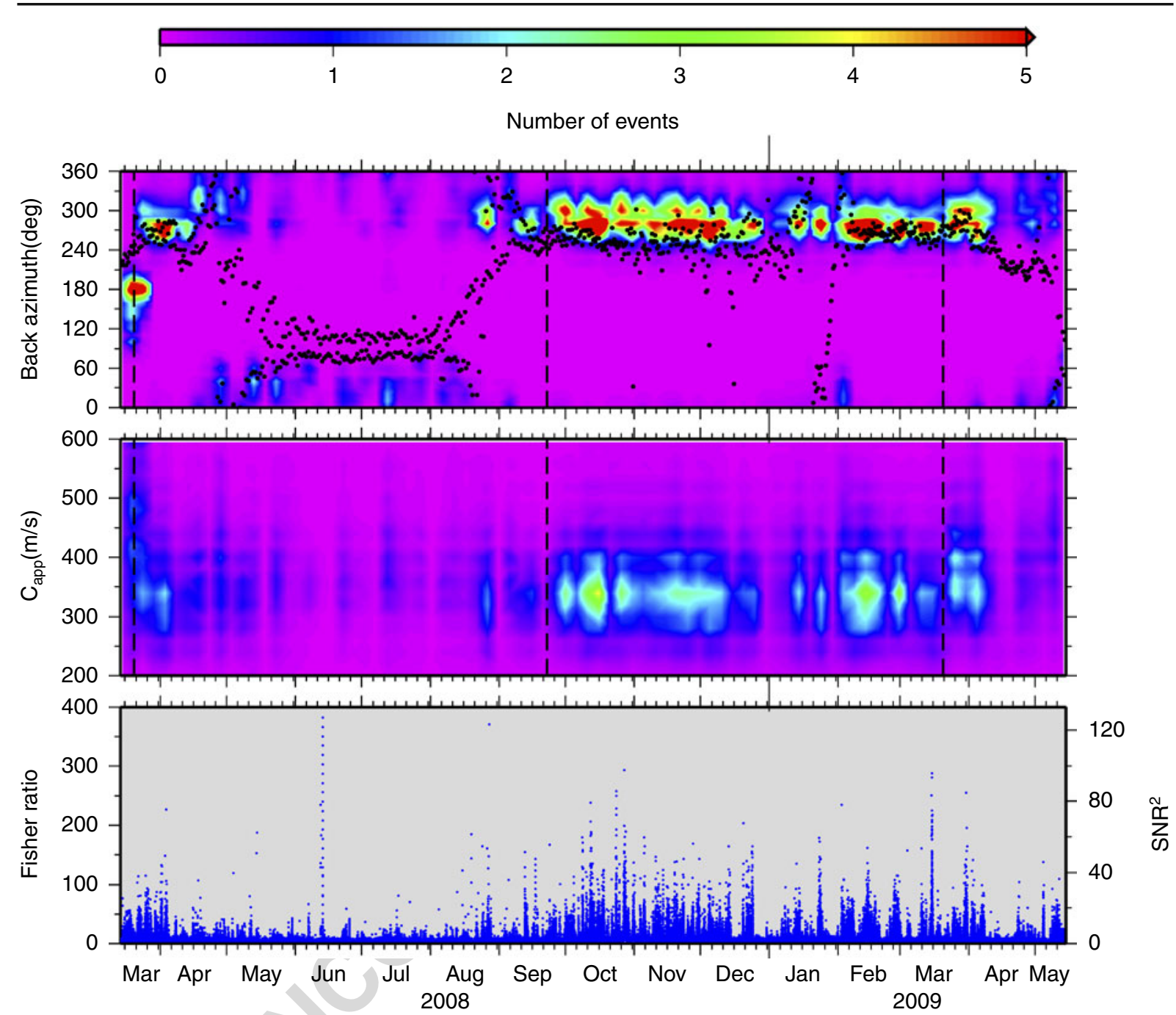

Fig. 5 Results from the array processing of ARCI data in the low-frequency band from 0.1 to $1.0 \mathrm{~Hz}$. The lower frame shows the Fisher ratios as function of time, that is, between March 13, 2008 and May 14, 2009. The Fisher ratio is related to the signal-to-noise (SNR) power ratio on the traces (see the axis on the right). The top frames

231 northeastern directions. In winter, events are de-

232 tected almost continuously and find their origin to 233 the west of ARCI.

234 Variations in the detectability of infrasound can 235 have several causes. These could be related to 236 the state of the atmosphere and variations of the 237 source (Le Pichon et al. 2009; Evers and Haak 238 2005). For the atmosphere, contributions along 239 the source-receiver path will be evaluated in the 240 following as well as near receiver effects. The gives the resolved apparent sound speed and backazimuth. Color coded are the number events per hour with a SNR larger than 1. Five or more events are indicated by red colors. The black dots represent the wind direction at $50 \mathrm{~km}$ altitude from the ECMWF analysis at $69.50 \mathrm{~N}, 25.50 \mathrm{E}$. The equinoxes are indicated by the vertical dashed lines

location, time, and strength of the source vary as 241 function of time and will also be analyzed.

\section{Contributions of the atmosphere}

4.1 General propagation characteristics

Atmospheric causes of the variations in the 245 detectability of infrasound are related to two 246 
247 distinct areas in the atmosphere, the stratosphere 248 and the boundary layer. The boundary layer is 249 approximately the first kilometer of atmosphere, 250 within the lower troposphere. The stratosphere 251 reaches from the tropopause, around $10 \mathrm{~km}$, up 252 to the stratopause near $50 \mathrm{~km}$ altitude. The ther253 mosphere, from $100 \mathrm{~km}$ and upwards, is not con254 sidered here because, in the considered frequency 255 range, thermospheric arrivals are strongly atten256 uated by the highly rarefied upper atmosphere. 257 These are, therefore, not expected to be observed 258 over ranges of over $1,000 \mathrm{~km}$ (Sutherland and 259 Bass 2004).

\subsection{Stratospheric variability}

261 The wind in the stratosphere, called the polar 262 vortex, varies on a seasonal scale. During win263 ter, winds are directed to the east, around the 264 stratopause on the Northern Hemisphere. These 265 winds can reach values of over $150 \mathrm{~m} / \mathrm{s}$. In sum266 mer, these winds are directed to the west and 267 somewhat less strong, reaching values of $70 \mathrm{~m} / \mathrm{s}$. 268 Figure 6 shows the wind and temperature near 269 ARCI, at $69.50 \mathrm{~N}, 25.50 \mathrm{E}$, as function of time. 270 These atmospheric specifications were obtained 271 from the European Centre for Medium-Range 272 Weather Forecasts (ECMWF). The wind is split 273 in a meridional and zonal component. The merid274 ional wind is the south-north component of the 275 wind and has a positive sign when directed to the 276 north. A positive sign for the zonal wind, which is 277 the west-east component, means it is directed to 278 the east. The change in the zonal wind direction 279 around the equinox should be noted, which causes 280 the anisotropy of the medium.

281 The temperature increase, due to presence of 282 ozone, and strong winds around $50 \mathrm{~km}$ altitude 283 may lead to bending of infrasonic waves back to 284 the Earth's surface, due to the increase in effective 285 propagation velocity. Changes in this so-called 286 stratospheric duct are visible in the surface based 287 microbarometer recordings of ARCI.

\section{4.2.1 Consequences for the high-frequency band}

289 For the high-frequency band, a distinction is made 290 between summer and winter in Fig. 4. It follows from this figure that events from the west are 291 more easily detected in winter as the stratospheric 292 winds are favorable for such propagation. Events 293 from the east are better detected in summer, but 294 some show up in wintertime. The detections of 295 sources which are not affected by the direction of 296 the polar vortex probably find their origin close to 297 the array, i.e., at distances less than $150 \mathrm{~km}$, where 298 tropospheric propagation is still dominant. 299

\subsubsection{Consequences for the low-frequency band}

In Fig. 5, the wind direction at $50 \mathrm{~km}$ altitude 301 is superimposed on the resolved backazimuths, 302 for the low frequency band. Clearly, the detec- 303 tion of coherent infrasound is guided by the 304 stratospheric wind. In winter, microbarom energy 305 from the Northern Atlantic Ocean is recorded. As 306 the winds turn around the equinox, microbarom 307 energy from the east is being detected. 308

As can been seen in Fig. 6, an abrupt change in 309 the winds and temperature occurred in the winter 310 of 2009, between late January and early Febru- 311 ary. Such changes are related to a major sudden 312 stratospheric warming (SSW; Holton 1979). The 313 temperature increases by $50^{\circ} \mathrm{C}$ in the stratosphere, 314 in only a couple of days, and the polar vortex 315 changes its direction. The major SSW also had 316 its effect on the infrasound detections (see also 317 Fig. 5). Suddenly, microbaroms from the east are 318 detected because of the change in direction of the 319 polar vortex, which is unusual in winter (Evers 320 and Siegmund 2009). 321

\subsection{Variability in the boundary layer}

The state of the boundary layer above the array 323 can cause de-correlation of the signals. A tur- 324 bulent atmosphere affects the signal coherency 325 which leads to a decrease of the detection capa- 326 bility. The summer boundary layer is far more 327 turbulent than the winter one. Heating of the 328 boundary layer due to solar radiation generates a 329 high degree of mixing. This effect is also visible 330 on a daily scale where the nighttime boundary 331 layer stabilizes as the influence of solar radiation 332 decreases.

Figure 7 shows the signal coherency, by means 334 of the Fisher ratio, for July and October 2008335 

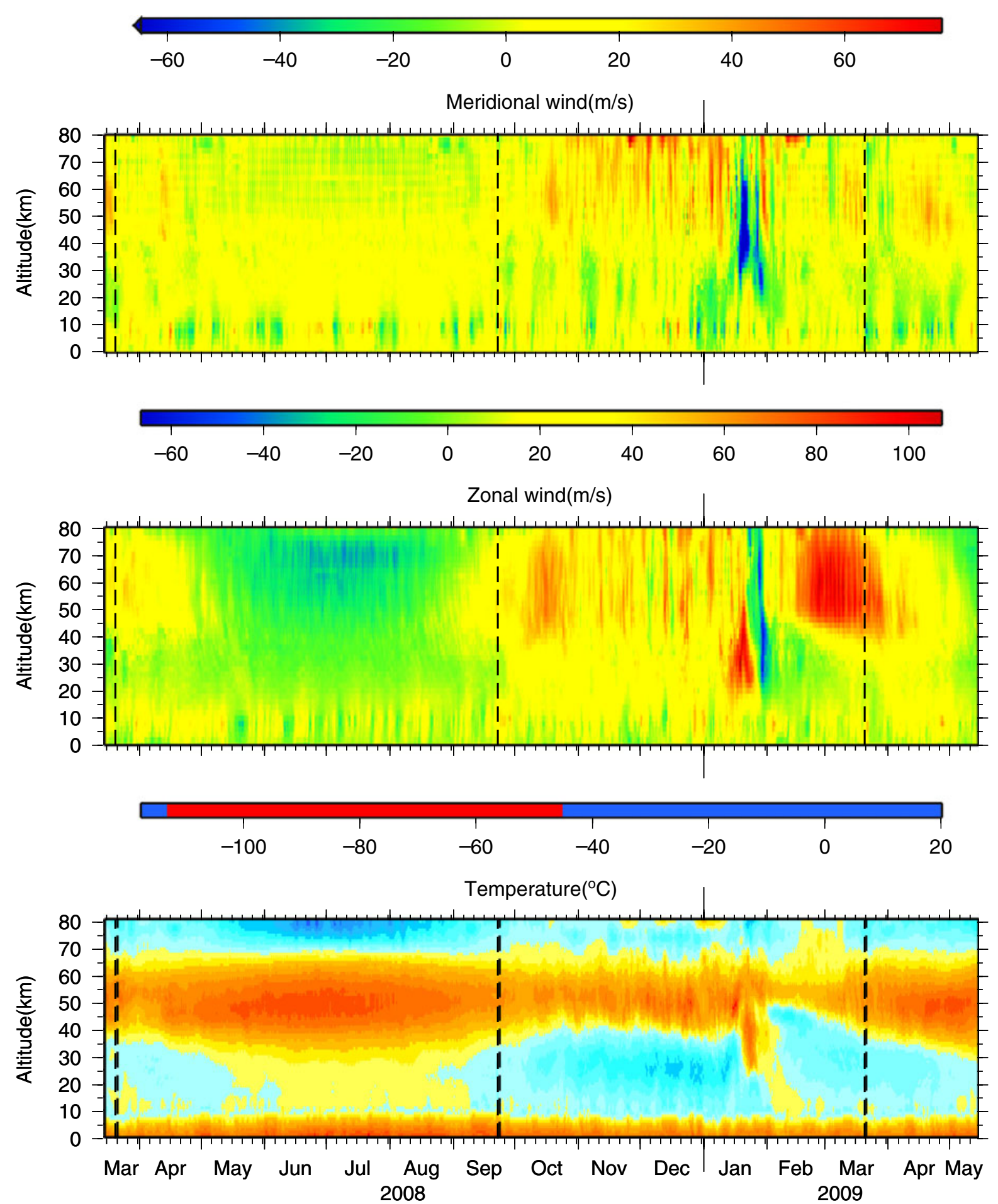

Fig. 6 The temperature and wind from the analyzes provided by the ECMWF. These models are available on a $0.5 \times 0.5^{\circ}$ grid, each $6 \mathrm{~h} /$ day. The grid node closest to ARCI is chosen, being $69.50 \mathrm{~N}, 25.50 \mathrm{E}$. The wind and temperature is modeled at 91 levels up to an altitude of approximately $80 \mathrm{~km}$. All values for the meridional wind lower than $-65 \mathrm{~m} / \mathrm{s}$ are colored blue, for plotting purposes, the actual lowest value is $-140 \mathrm{~m} / \mathrm{s}$. The equinoxes are indicated by the vertical dashed lines 

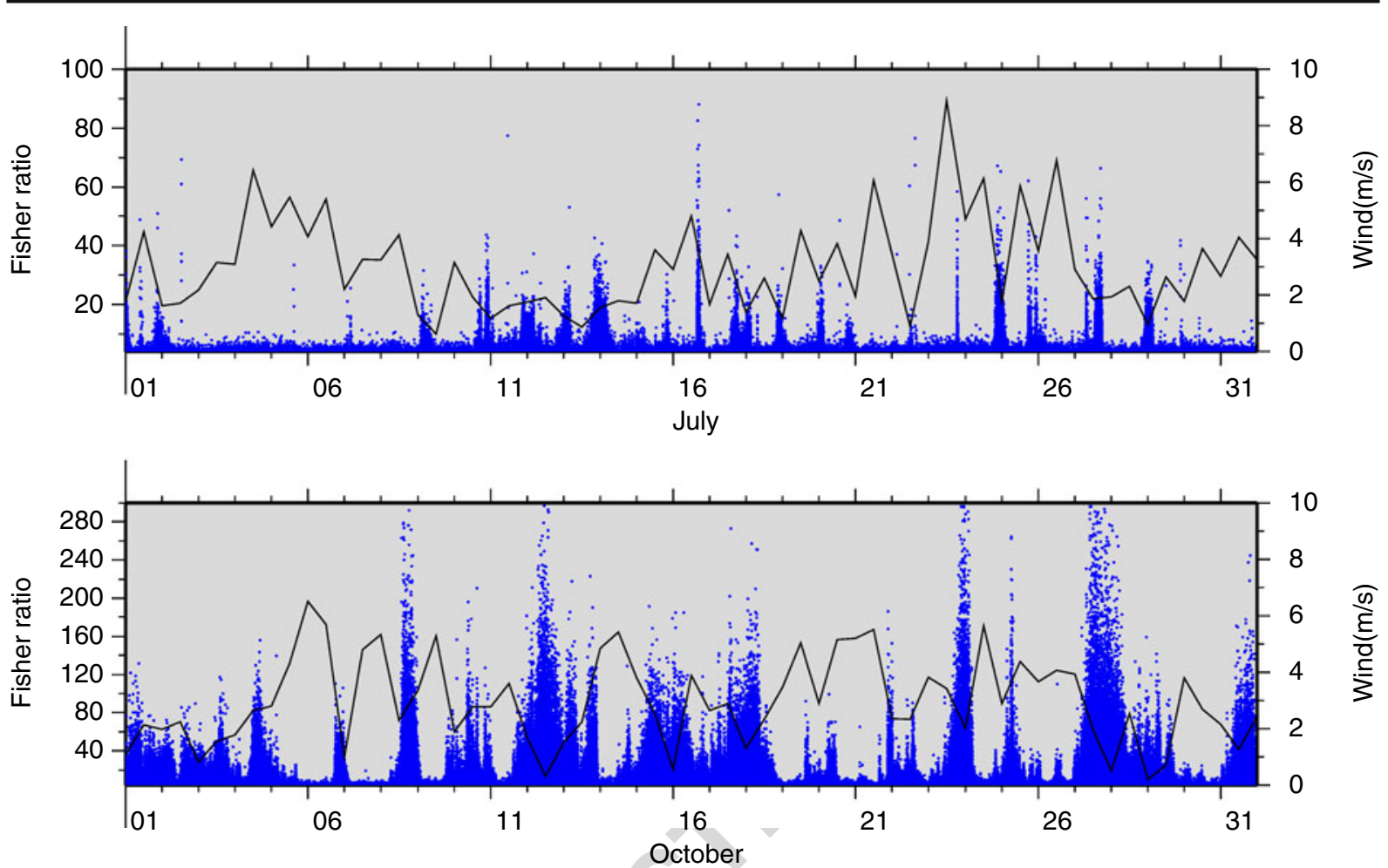

Fig. 7 The Fisher ratios for July (top) and October 2008 (bottom frame), for the low-frequency band. Superimposed are the wind strengths, as solid black lines, at the first

336 in the low-frequency band. Superimposed are the 337 wind strengths from ECMWF models, at 69.50 $338 \mathrm{~N}, 25.50 \mathrm{E}$, for the first level which is slightly, 339 i.e., around $300 \mathrm{~m}$, above the Earth's surface. It 340 follows from this figure that the wind strength in 341 summer varies on a daily basis. It peaks during 342 daytime and decreases at night when the influence 343 of solar radiation is reduced. The reduction in 344 wind leads to an increase in the detectability of in345 frasound which is reflected by higher Fisher ratios. 346 Wind variations in winter have longer periods, but 347 also here an increase in wind leads to a decrease in 348 performance of the array.

\section{Specifications of the microbarom sources}

\section{5.1 Description of microbarom source}

351 The source generating the signals, in the low352 frequency band, varies in strength over time. The level of the ECMWF models at $69.50 \mathrm{~N}, 25.5 \mathrm{E}$. This first level corresponds to an altitude slightly above the Earth's surface microbaroms are generated by the non-linear in- 353 teraction of oceanic waves, which often occurs 354 in the vicinity of low-pressure systems over the 355 oceans. The interference of almost oppositely 356 traveling waves leads to pressure signals in both 357 the atmosphere and the solid Earth, i.e., micro- 358 seism. The signals have a dominant frequency 359 around $0.2 \mathrm{~Hz}$, which is double the frequency 360 of the oceanic waves. The amplitude of induced 361 pressure waves $\left(I_{\mathrm{S}}\right)$ is, in first order, propor- 362 tional to the squared multiplication of the wave 363 height $(a)$ and frequency $(\omega)$, thus $I_{\mathrm{S}} \sim(a \omega)^{2} 364$ (Posmentier 1967). To accurately predict the gen- 365 eration of microbaroms, the directional spectra of 366 oceanic waves should be evaluated to identify the 367 almost oppositely traveling waves and their peri- 368 ods (Kedar et al. 2008). Here, it is assumed that 369 the waves are interacting near the maximum of 370 the squared multiplication of the wave height and 371 frequency. This allows for an efficient calculation, 372 to get an indication of the source activity (Evers 373 
374 and Haak 2001). An independent approach will 375 also be tested where the occurrence of micro376 seism in the seismic recordings of ARCES are 377 evaluated.

3785.2 Wave height and frequency from oceanic 379 wave models

380 Figure 8 shows the backazimuths in the direction 381 of microbarom activity in the Atlantic and Pacific
Ocean, from 12-hourly oceanic wave models pro- 382 vided by the ECMWF. The source intensity, $I_{\mathrm{S}}$, is 383 also estimated. The observed backazimuths of the 384 infrasound and direction of microbarom activity 385 coincide throughout the seasons. The detection of 386 microbaroms is also clearly related to the direc- 387 tion of the stratospheric winds (Garcés et al. 2004; 388 Le Pichon et al. 2006). During the SSW which 389 occurred in the winter of 2009, there is a sudden 390 change in resolved backazimuths. Microbarom en- 391 ergy from the Pacific Ocean is detected, during 392

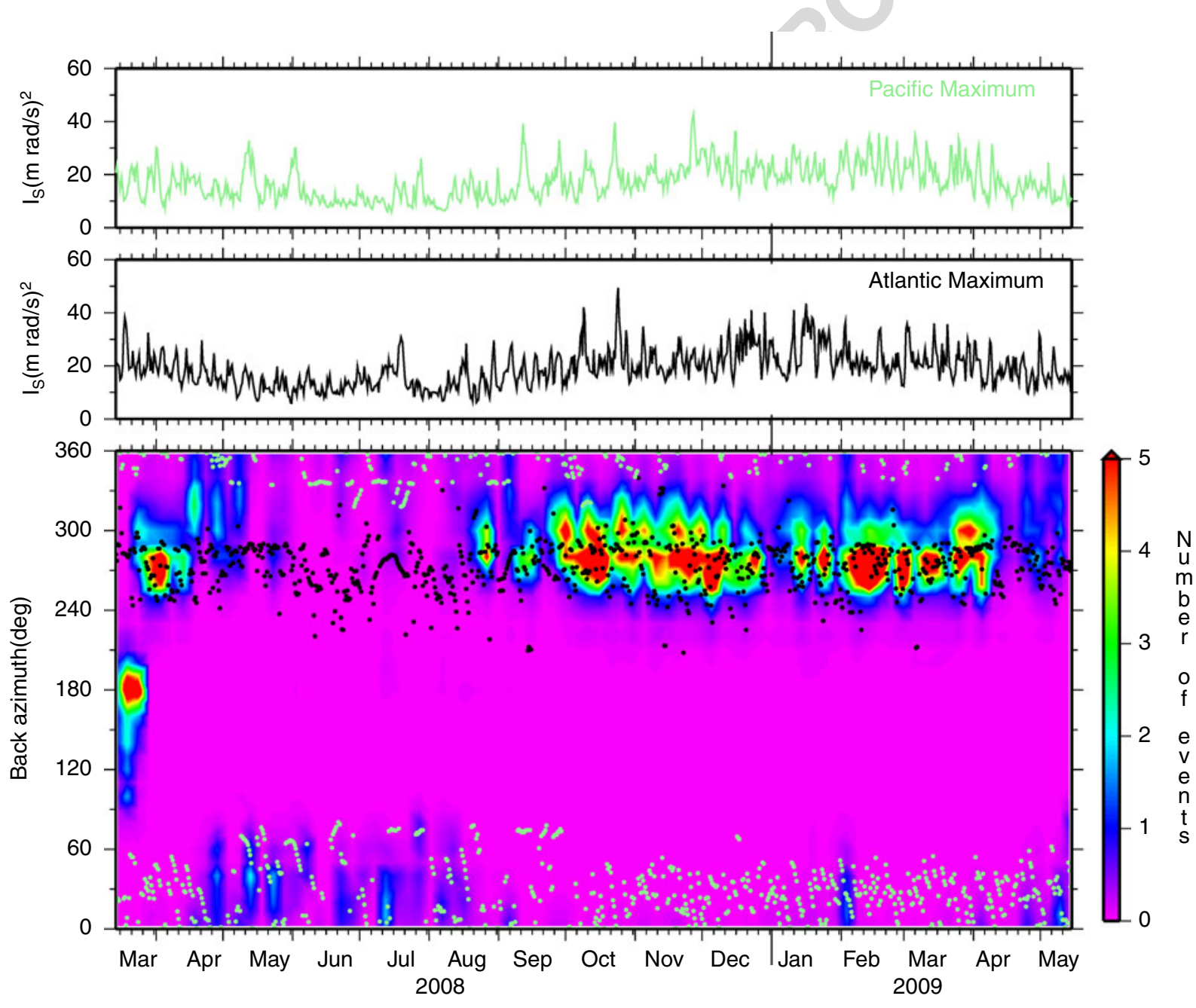

Fig. 8 An estimate of the microbarom activity in the Atlantic (black dots) and Pacific Ocean (green dots). The dots give the directions, i.e., backazimuths, to the Atlantic and Pacific maxima. The retrieved directions, in the lower frame, and source intensities ( $I_{\mathrm{S}}$, in the upper frames) are calculated from 12-hourly oceanic wave models from ECMWF provided at each $0.5 \times 0.5^{\circ}$ 


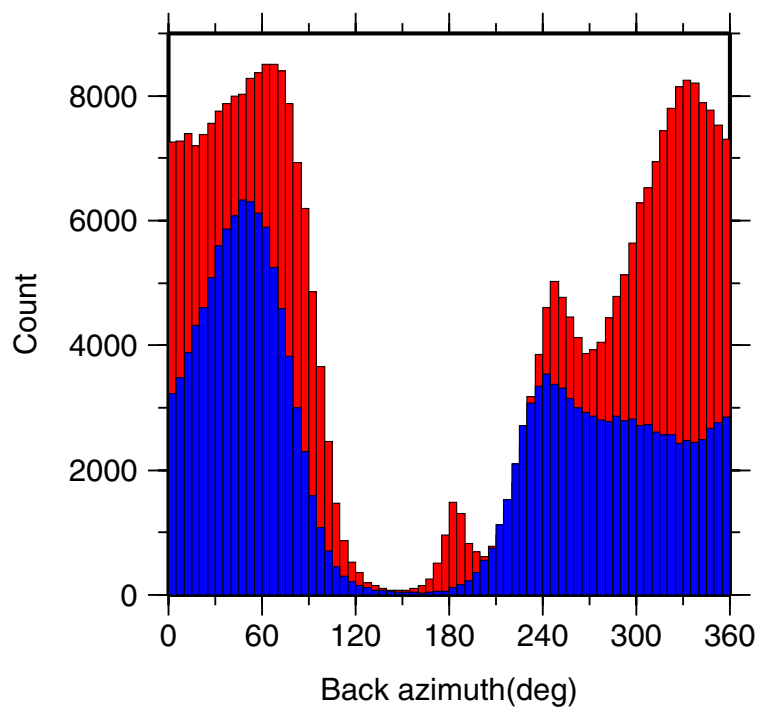

Fig. 9 Detections of microseism at the ARCES array. These detections are split in summer (in red, April through September) and winter (blue) a short period in early February. This indicates 394 that the low-frequency energy detected during 395 summer might also find its origin on the Pacific 396 Ocean.
5.3 Comparison with microseismic detections

Seismic data from the ARCES array (see Fig. 1) 398 are processed to detect energy from microseism. 399 The processing sequence is as follows:

- Band-pass filter with a third-order Butter- 401 worth filter with corner frequencies of 0.1 and 402 $0.4 \mathrm{~Hz}$.

- Decimate the data with a factor of 8 , from a 404 40- to 5-Hz sampling rate. $\quad 405$

- Split the data in a window of $20 \mathrm{~s} . \quad 406$

- Apply a frequency-wavenumber analysis, be- 407 tween 0.15 and $0.25 \mathrm{~Hz}$, by moving this win- 408 dow with $6 \mathrm{~s}$.

- Calculate a beam and measure the maximum 410 amplitude in the window.

The array response of ARCES for a $0.2-\mathrm{Hz} 412$ planar wave is given in Fig. 2. At such low frequen- 413 cies, the main lob is quite broad but its maximum 414 can still be determined with enough accuracy for 415 this study, since only a rough estimate $\left( \pm 5^{\circ}\right)$ of 416 the backazimuth is sufficient. The detections of 417 microseism at ARCES are shown in Fig. 9. A 418 total of 6.4 million coherent seismic arrivals are 419 detected between 0.15 and $0.25 \mathrm{~Hz}$. This number is 420

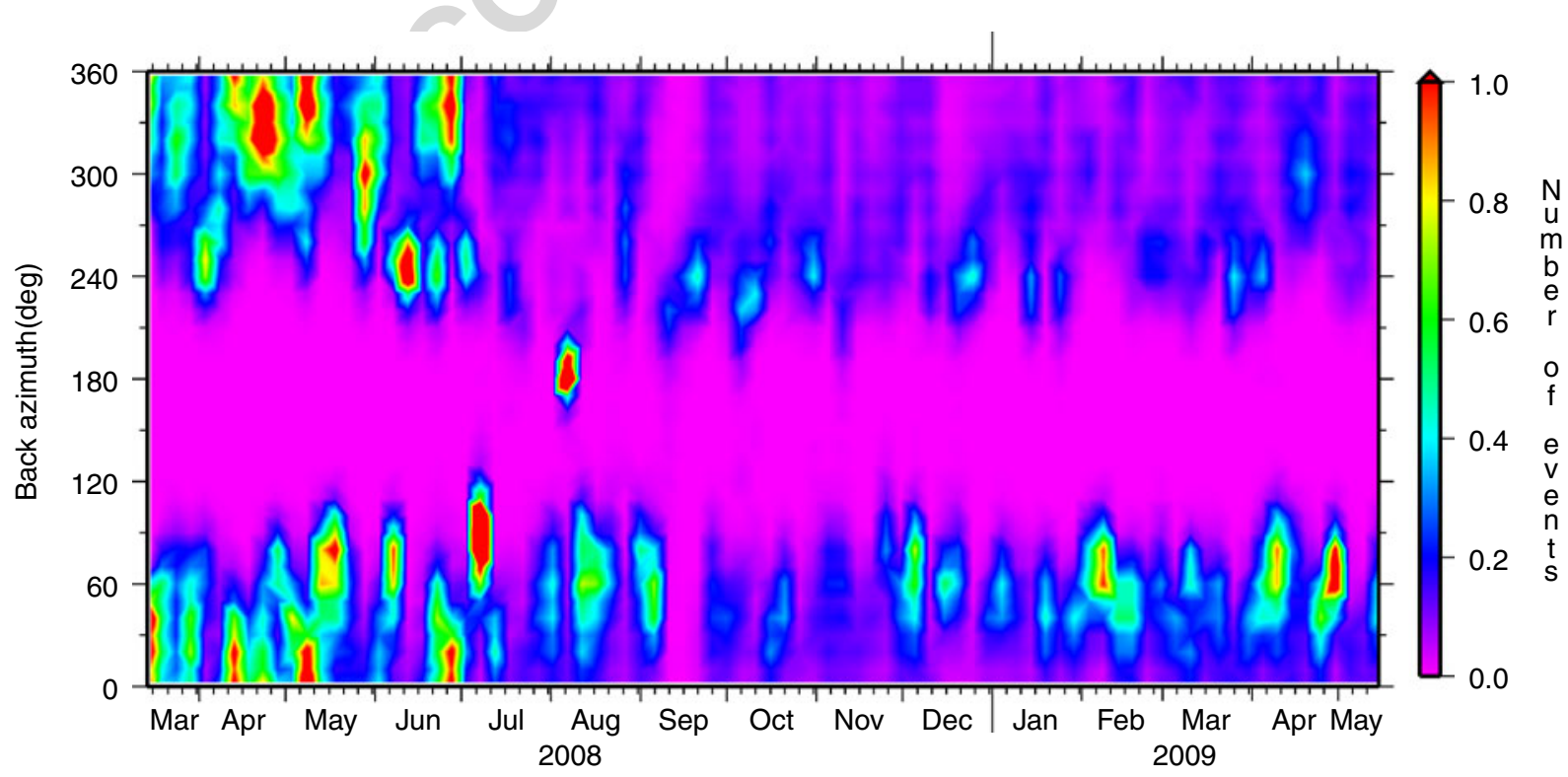

Fig. 10 The occurrence of microseism at the ARCES array. Detections are contoured as function of time and backazimuth. The detections are averaged per hour and one or more detections are indicated by red colors 
449 Infrasound data from ARCI have been processed 450 by evaluating the Fisher ratio over the period 451 of March 13, 2008 up to May 14, 2009. With a 452 detection threshold at a SNR of 1, 1.8 million 453 events are detected between 0.1 and $1.0 \mathrm{~Hz}$ and 45416,475 events between 1.0 and 7.0 Hz. Detections 455 in the low-frequency band are mostly related to 456 the interaction of oceanic waves which leads to 457 microbaroms. In the high-frequency band, mainly 458 man-made events are detected which are related 459 to mining and military activity. Similar findings 460 have been reported by Le Pichon et al. (2008).

461 The characteristics of the medium, i.e., wind 462 and temperature structure up to stratospheric al463 titudes, and the source have been derived from 464 ECMWF models. A clear relation has been shown 465 between upper atmospheric winds and the direc466 tionality of the detections for the low-frequency band. These seasonal changes are also partly vis- 467 ible in the high-frequency band. In winter, the 468 sources to the west are detected while preference 469 is given to sources in the east during summer. 470 The state of the boundary layer, or turbulence 471 and low-level winds, partly determines the signal 472 coherency. In summer, there is a daily variation 473 caused by the influence of solar radiation. A more 474 stable boundary layer during nighttime leads to 475 less coherency loss. $\quad 476$

In addition, microbarom activity has been esti- 477 mated by evaluating the ocean wave height and 478 period. ARCI is sensitive to microbaroms from 479 the Atlantic Ocean in winter. Microbarom energy 480 from the east is detected during summer. This 481 anisotropic behavior was also identified during a 482 period of only a couple of days, related to a SSW. 483 A sudden change was noted from the detection 484 of microbarom energy from the Atlantic Ocean to 485 those from the Pacific Ocean. 486

The importance of taking into account both 487 the characteristics of the medium and the source 488 is illustrated by comparing Figs. 5 and 8 . The 489 detections move from west $\left(270^{\circ}\right)$ to northwest 490 $\left(330^{\circ}\right)$ during March and April 2008. It follows 491 from Fig. 8 that the sources, microbaroms in the 492 Atlantic Ocean, are occurring with a more or less 493 stable backazimuth between $270^{\circ}$ and $300^{\circ}$. The 494 stratospheric wind, on other hand, is varying from 495 southwest to north during this period. Therefore, 496 this change in the resolved backazimuths should 497 be attributed to the wind which enables the detec- 498 tion of an unknown source to the south of ARCI. 499

Another change is visible, in Fig. 8, between 500 October 2008 and April 2009. The resolved back- 501 azimuths tend to move somewhat from the north- 502 west to the west. The cause should be related to 503 the source, as the wind shows no evidence for 504 such translation. Whether this change relates to 505 the southward movement of sea ice during winter 506 remains to be investigated. It is hypothesized that 507 the sea ice blocks the northward propagation of 508 oceanic waves. Consequently, the generation of 509 microbaroms is limited up to a certain longitude. 510 This is also indicated by the microseism detec- 511 tions. The source seems limited in its northward 512 propagation during winter (see Fig. 10).

The seasonal variations in microbarom detec- 514 tions also follow from the comparison with the 515 
516 microseismic detections. The highly dynamic and 517 anisotropic nature of the atmosphere can prohibit 518 the detection of energy from certain directions. 519 The ocean wave activity, i.e., generation of mi520 croseism, is almost continuously present from the 521 Atlantic and Pacific Ocean. The microbaroms ap522 pear from $270^{\circ}$ during winter, while the micro523 seism have a dominant backazimuth of $240^{\circ}$. The 524 latter direction coincides with the location found 525 by Essen et al. (2003) which was just off coast 526 of Norway. The microbaroms are probably gen527 erated in the deep ocean as the direction points to 528 a location similar to the one found by Evers and 529 Haak (2001) and Kedar et al. (2008), which was in 530 a region to the south of Greenland and Iceland. 531 Further research will be carried out to determine 532 the origin of the microseism and correlate those to 533 microbaroms. Better statistics will be obtained by 534 evaluating more than 1 year of data, by excluding, 535 for example, special weather conditions.

536 In conclusion, the general behavior of an in537 frasound array, like ARCI, can be understood 538 by evaluating the detectability in relation to at539 mospheric processes and source activity. Upper 540 atmospheric winds and the state of the boundary 541 layer play an important role in the detectability 542 of infrasound. Understanding such dependencies 543 is important for the identification of small-sized 544 nuclear test which are expected to occur in the 545 low-frequency or microbarom band.

546 Acknowledgements The work performed in this study 547 was supported by a Transnational Access (TA) grant. This 548 TA grant was provided by NERIES, an EC project with 549 contract number RII3-CT-2006-026130. Figures in this ar550 ticle were made with the Generic Mapping Tools (Wessel 551 and Smith 1991).

\section{References}

553 Balachandran NM, Donn WL, Rind D (1977) Concorde sonic booms as an atmospheric probe. Science 197:4749

Dahlman O, Mykkeltveit S, Haak H (2009) Nuclear test ban. Springer, Dordrecht

Drob DP, Picone JM, Garcés MA (2003) The global morphology of infrasound propagation. J Geophys Res 108:4680

Essen HH, Krüger F, Dahm T, Grevemeyer I (2003) On the generation of secondary microseisms observed in northern and central Europe. J Geophys Res 108:2506
Evers LG, Haak HW (2001) Listening to sounds from an 564 exploding meteor and oceanic waves. Geophys Res 565 Lett 28:41-44 566

Evers LG, Haak HW (2005) The detectability of infra- 567 sound in the Netherlands from the Italian volcano Mt. 568 Etna. J Atmos Sol-Terr Phys 67:259-268 569

Evers LG, Siegmund P (2009) Infrasonic signature of the 570 2009 major sudden stratospheric warming. Geophys 571 Res Lett 23:L23,808 572

Garcés MA, Willis M, Hetzer C, Le Pichon A, Drob D 573 (2004) On using ocean swells for continuous infra- 574 sonic measurements of winds and temperature in the 575 lower, middle, and upper atmosphere. Geophys Res 576 Lett 31:L19,304 577

Gossard EE, Hooke WH (1975) Waves in the atmosphere. 578 Elsevier Scientific, Amsterdam 579

Hedlin MAH, Alcoverro B, D’Spain G (2003) Evaluation 580 of rosette infrasonic noise-reducing spatial filters. J 581 Acoust Soc Am 114:1807-1820 582

Holton JR (1979) An introduction to dynamic meteorol- 583 ogy. Academic, London 584

Kedar SM, Longuet-Higgins MS, Webb F, Graham N, 585 Clayton R, Jones C (2008) The origin of deep ocean 586 microseisms in the North Atlantic Ocean. Proc R Soc 587 Lond A 464:777-793 588

Le Pichon A, Ceranna L, Garés M, Drob D, Millet C (2006) 589 On using infrasound from interacting ocean swells 590 for global continuous measurements of winds and 591 temperature in the stratosphere. J Geophys Res 111: 592 D11,106 593

Le Pichon A, Vergoz J, Herry P, Ceranna (2008) Analyzing 594 the detection capability of infrasound arrays in central 595 Europe. J Geophys Res 113:D12,115 596

Le Pichon A, Vergoz J, Blanc E, Guilbert J, Ceranna L, 597 Evers LG, Brachet N (2009) Assessing the perfor- 598 mance of the international monitoring system infra- 599 sound network: geographical coverage and temporal 600 variabilities. J Geophys Res 114:D8112 601

Liszka L (1978) Long-distance focusing of concorde sonic 602 boom. J Acoust Soc Am 64:631-635 603

Melton BS, Bailey LF (1957) Multiple signal correlators. 604 Geophysics XXII:565-588 605

Posey JW, Pierce AD (1971) Estimation of nuclear explo- 606 sion energies from microbarograph records. Nature 607 232:253 608

Posmentier ES (1967) A theory of microbaroms. Geophys 609 J R Astron Soc 13:487-501 610

Roth M, Fyen J, Larsen PW (2008) Setup of an experimen- 611 tal infrasound deployment within the ARCES array. 612 Scientific Report 2-2008, NORSAR 613

Smart E, Flinn EA (1971) Fast frequency-wavenumber 614 analysis and Fisher signal detection in real-time infra- 615 sonic array data processing. Geophys J R Astron Soc 616 26:279-284

Sutherland LC, Bass HE (2004) Atmospheric absorption 618 in the atmosphere up to $160 \mathrm{~km}$. J Acoust Soc Am 619 115:1012-1032 620

Symons GJ (ed) (1888) The eruption of Krakatoa and sub- 621 sequent phenomena. Trübner \& Co., London 622

Wessel P, Smith WHF (1991) Free software helps map and 623 display data. EOS Trans AGU 72:441 
625 Whipple FJW (1930) The great Siberian meteor and the 626 waves, seismic and aerial, which it produced. Q J R Meteorol Soc 56:287-304
Whipple FJW (1939) The upper atmosphere, density and 628 temperature, direct measurements and sound evi- 629 dence. Q J R Meteorol Soc 65:319-323 


\section{AUTHOR QUERY}

\section{AUTHOR PLEASE ANSWER QUERY}

Q1. Figures 5-8 were rasterized. Please check if captured appropriately. 